\title{
Efficacy and safety of a lidocaine/tetracaine medicated patch or peel for dermatologic procedures: a meta-analysis
}

\author{
Won Oak Kim, Byung Min Song, and Hae Keum Kil \\ Department of Anesthesiology and Pain Medicine, Anesthesia and Pain Research Institute, Yonsei University College of Medicine, \\ Seoul, Korea
}

Background: To justify the use of the lidocaine/tetracaine medicated patch or peel as a preventive treatment for reducing pain and discomfort in adults and children. We reviewed randomized controlled trials (RCTs) to evaluate the efficacy and safety of the lidocaine/tetracaine medicated patch or peel compared with placebo.

Methods: Ten RCTs (574 patients) were included in this systemic review. Relevant studies were identified through searches of MEDLINE, SCOPUS and the Cochrane database library. The outcome was the adequacy of cutaneous anesthesia reflected in the patient's assessment of pain intensity during minor dermatologic procedures and adverse effects after application of the lidocaine/tetracaine medicated patch or peel versus placebo.

Results: The efficacy of the lidocaine/tetracaine patch or peel was consistently very significantly beneficial 30 or 60 minutes after the application compared to placebo (Relative risk, RR: 2.5; Number needed to treat, NNT: 2.2). We did not identify any difference in the effectiveness of adequate analgesia between the lidocaine/tetracaine patch and peel (the number needed to treat or to harm, NNT 2.4 vs. 2.0). No serious side effects or adverse events were observed with the lidocaine/tetracaine medicated patch or peel and placebo. Minor skin reactions were transient and resolved without treatment (Odd ratio, OR: 1.4 and 95\% confidence interval, CI: 0.9-2.1; NNT: 14.9).

Conclusions: The lidocaine/tetracaine medicated patch or peel is a well accepted, effective and safe method for minor dermatologic procedures based on pooled data of trials in terms of adequacy of cutaneous anesthesia and adverse effects. (Korean J Anesthesiol 2012; 62: 435-440)

Key Words: Lidocaine, Meta-analysis, Tetracaine, Topical anesthetics.

Received: May 20, 2011. Revised: 1st, June 13, 2011; 2nd, June 21, 2011; 3rd, June 22, 2011. Accepted: June 24, 2011.

Corresponding author: Won Oak Kim, M.D., Department of Anesthesiology and Pain Medicine, Anesthesia and Pain Research Institute, Yonsei University College of Medicine, 134, Shinchon-dong, Seodaemun-gu, Seoul 120-752, Korea. Tel: 82-2-2228-2413, Fax: 82-2-312-7185, E-mail: wokim@yuhs.ac

(c) This is an open-access article distributed under the terms of the Creative Commons Attribution Non-Commercial License (http:// creativecommons.org/licenses/by-nc/3.0/), which permits unrestricted non-commercial use, distribution, and reproduction in any medium, provided the original work is properly cited. 


\section{Introduction}

Pain and discomfort following minor dermatologic procedures are often underestimated and can be stressful for patients. Relieving or preventing such distress is a serious clinical concern, especially in children. There are several available topical local anesthetics for various dermatologic procedures which may be associated with pain or discomfort. Hence, topical anesthetics which significantly prevent pain and discomfort can be used for minor superficial procedures instead of general anesthesia or needle injection of local anesthetics. Consequently, even children can better tolerate the procedures with lessened anxiety and distress by effective pain management. Proper control of pain and discomfort is needed to relieve patients' suffering and produce better outcomes $[1,2]$.

An ideal local anesthetic should be effective, painless cutaneous analgesia with a short time of onset, have sufficient duration but not have untoward effects. Various options have been attempted and developed to overcome the barrier of the stratum conium for rapid and effective drug delivery with minimal systemic absorption and adverse effects. The options include topical application or injection, iontophoresis, sonophoresis, laser-assisted transdermal passage, pressurized gas delivery and heat-enhanced diffusion [3]. Topical application of local anesthetics is currently considered to be the easiest, most effective and convenient way for treatment of patients who may be undergoing superficial dermal procedures. The most commonly used dermal analgesics are lidocaine, tetracaine, prilocaine or combinations thereof [3-5].

A lidocaine/tetracaine patch $\left(\right.$ Synera $^{\mathrm{TM}}$ ) for topical anesthesia as a eutectic $1: 1$ mixture with an oxygen-activated heating element has been introduced. This novel drug delivery system was developed to warm the skin and improve the delivery of local anesthetics through the skin. The same formula was designed as a local anesthetic peel (S-Caine Peel) which is a pliable peel with a skin-like appearance when exposed to air and becomes an easily removable flexible membrane. Clinical studies demonstrated that the S-Caine Patch or Peel is efficacious in relieving the pain associated with superficial dermal procedures $[6,7]$.

The objective of this meta-analysis was to examine outcomes such as adequate analgesic efficacy and safety after application of the lidocaine/tetracaine medicated patch or peel in published randomized controlled trials (RCTs). We identified a considerable number of clinical studies from several databases which explored the efficacy and safety of the lidocaine/tetracaine medicated patch or peel as a local anesthetic for minor dermatologic procedures and minimally invasive cutaneous procedures. This report extensively reviewed the literature and evaluated the pharmacologic efficacy and safety of the patch and peel through the primary outcome of pain based on the patient assessment. We found a substantial number of published RCTs of the lidocaine/tetracaine patch or peel used in different dermatologic procedures and selected these according to the method described below. Quantitative methods were used to summarize the results, where possible.

\section{Materials and Methods}

A systemic search of the relevant literature was performed without language limitations. We mainly searched MEDLINE using PUBMED, SCOPUS and the Cochrane database library as well as reference lists of reviews or initially identified articles which were also used for further articles related to the topic $[2,4-6,8,9]$.The search was performed with key words of "topical anesthetics", "s-caine", "synera" and "lidocaine/tetracaine" in their titles or abstracts and electronic searches were conducted until October 2010. Inclusion criteria were: (1) prospective RCT with full reports on the efficacy and safety of the lidocaine/ tetracaine medicated patch or peel compared with placebo; (2) placebo was identical in appearance and had no active drugs, and contained the same heating element in the case of patch application; (3) investigation was performed in humans (adults and children); (4) the measured outcomes (after applying the patch or peel 30 or 60 minutes prior to procedures) included adequacy of cutaneous anesthesia as well as adverse effects after application of the lidocaine/tetracaine medicated patch or peel; (5) the patient's assessment of pain intensity during the minor dermatologic procedures such as needle insertion, laser treatment and IV cannulation, etc. We did not include data from abstracts, posters, case reports, letters to the editor, reviews or animal research. Only prospective randomized, double-blinded, placebo controlled trials that evaluated the efficacy and safety of the lidocaine/tetracaine medicated patch or peel were included. More invasive procedures involving skin incision, graft and trials investigating topical application of mucous membrane and open wounds were excluded. No attempts were made to obtain unpublished studies or did we request for unpublished data from any company developing related drugs.

Selected reports were examined and scored using the fivepoint Oxford scale for assessment of validity by one of the authors $[1,10]$. Collected data were recorded on formalized sheets and we converted the adequacy of anesthesia originally presented as a percentage of the number of patients for analysis, where needed. We computed relative benefits (RB) as relative risks (RR) of the lidocaine/tetracaine medicated patch or peel with $95 \%$ confidence intervals (CI). We calculated the number needed to treat or to harm (NNT) as a useful estimated measure of a significant clinical effect. NNT is the number of 
patients that must be treated with an experimental intervention (the lidocaine/tetracaine medicated patch or peel) to achieve a particular result (beneficial or harmful) in one of them which would not have been the case had they all received the control interventions (placebo). Many studies on adverse effects had zero cells (i.e., trials had no report on any event in one of the study groups) that odds ratio (OR) with $95 \%$ confidence intervals was presented. Statistical analysis was performed using MIX 1.7 for Windows that was developed and validated by Leon Bax of the Department of Medical Informatics of Kitasato University in Japan $[11,12]$.

\section{Results}

We identified 16 randomized controlled trials and included 10 of them in the present systemic review, thereby enrolling a total 574 subjects [7,13-23]. Among the six other studies, one article had no accessible dichotomous data on safety and efficacy with only P values [20]. Five other studies compared S-caine and EMLA or $2 \%$ lidocaine, which was an inappropriate placebo for our review, and one of them tested the contribution of a heating element to the topical patch [14,24-27]. A detailed summary of each trial, including methodological quality scores, is presented in Table 1 . The median quality of score was 3 in two trials, 4 in two and 3 in six. Two papers which scored 5 reported appropriate description of randomizations (i.e., computer-generated or table of random number) and blinding (i.e., an active and indistinguishable placebos were used) $[7,23]$. Most trials used VAS to measure the adequacy of anesthesia. Surrogate pain scores provided by investigators, parents or observers were not included in the analysis.

Data were pooled for calculation and computed for combining analysis. All trials showed positive effects for the adequacy of cutaneous anesthesia (Fig. 1). Cochran Q statistic $P$ value (0.4) was above 0.05 , demonstrating homogeneity in the trials. As a result, a fixed-effects model with a weighting method (inverse variance) developed by DerSimonian and Laird was used. Relative risk (relative benefit) was 2.5 (95\% confidence interval 2.0-3.1) and efficacy of cutaneous anesthesia by the lidocaine/tetracaine medicated patch or peel compared to placebo was highly significant $(\mathrm{P}<0.01$ : Fig. 1$)$. NNT for effectiveness of adequacy of anesthesia was calculated as 2.2. Subgroup analysis results are shown in Table 2. Subgroups were similar with each other in terms of homogeneity, effects model and NNT. They consistently showed highly significant benefits of the lidocaine/tetracaine medicated patch or peel compared to placebo.

Eight trials reported on adverse effects which included erythema, burning sensation, itching, blisters and edema in both groups [13,16-19,21-23]. No weighting was used for different grades of adverse effects. Mild erythema was the most often observed adverse effect, but all adverse events were transient and resolved without treatment. Slight blanching was noted but no delayed allergic skin reactions were observed.

Table 1. Summary of Randomized, Double-Blinded, Placebo-Controlled Trials

\begin{tabular}{|c|c|c|c|c|c|c|c|c|c|c|c|}
\hline Study ID & $\begin{array}{l}\text { Quality } \\
\text { score }\end{array}$ & Age range & $\begin{array}{l}\text { Application } \\
\text { time, min. } \\
\text { (type) }\end{array}$ & Intervention & $\begin{array}{l}\text { Measurement } \\
\text { of pain }\end{array}$ & $\begin{array}{c}\text { Total } \\
\text { no. }\end{array}$ & $\begin{array}{c}\text { Active } \\
\text { drug } \\
\text { ACA, no. }\end{array}$ & $\mathrm{AR}$ & $\begin{array}{c}\text { Total } \\
\text { no. }\end{array}$ & $\begin{array}{c}\text { Placebo } \\
\text { ACA, } \\
\text { no. }\end{array}$ & $\mathrm{AR}$ \\
\hline Shomaker (2000) & 5 & $19-36$ & 30 (patch) & Needle insertion & Verbal scale $(0-2)$ & 12 & 11 & 0 & 12 & 0 & 1 \\
\hline Bryan (2002) & 3 & $27-56$ & 30 (peel) & Laser Tx(vascular lesion) & VAS & 10 & 10 & 6 & 5 & 0 & 4 \\
\hline Chen (2003) & 3 & $>18$ & 60 (peel) & Laser Tx (leg vein) & VAS & 20 & 15 & 0 & 20 & 7 & 0 \\
\hline Doshi(2003) & 3 & 49 (mean) & 30 (peel) & Laser Tx (cheek) & VAS & 20 & 18 & 1 & 20 & 3 & 0 \\
\hline $\operatorname{Jih}(2004)$ & 3 & $22-72$ & 60 (peel) & Laser Tx (leg vein) & VAS & 60 & 40 & 38 & 60 & 16 & 35 \\
\hline Sethna (2005) & 4 & $3-17$ & 20 (patch) & Vascular access & Oucher scale & 41 & 24 & $22(43) *$ & 20 & 4 & $11(21)^{*}$ \\
\hline Chen (2005) & 3 & $21-43$ & 60 (peel) & Laser Tx (tatoo) & VAS & 30 & 16 & 20 & 30 & 3 & 14 \\
\hline Berman (2005) & 3 & $20-80$ & 30 (patch) & Minor procedures & VAS & 45 & 33 & 1 & 49 & 18 & 0 \\
\hline Curry (2007) & 4 & $21-61$ & 20 (patch) & Vascular access & VAS & 40 & 29 & 2 & 40 & 12 & 0 \\
\hline Singer (2008) & 5 & $3-17$ & $\begin{array}{l}39 \text { (patch), } \\
33 \text { (placebo) }\end{array}$ & IV cannulation & VAS & 20 & 15 & 6 & 20 & 7 & 6 \\
\hline
\end{tabular}

ACA: Adequacy of cutaneous anesthesia, AR: Adverse skin reaction, Tx: Treatment. *Different patient's number for skin reaction.

Table 2. Pooled and Separated Analysis of Effects Based on Application Type

\begin{tabular}{lcccc}
\hline Data & Q statistics & Effects model & RR-outcome (95\% CI) & NNT \\
\hline Pooled & $\mathrm{P}=0.4$ & Fixed effects model $(\mathrm{P}<0.01)$ & $2.5(2.0-3.1)$ & 2.2 \\
Patch & $\mathrm{P}=0.5$ & Fixed effects model $(\mathrm{P}<0.01)$ & $2.3(1.7-3.0)$ & 2.4 \\
Peel & $\mathrm{P}=0.3$ & Fixed effects model $(\mathrm{P}<0.01)$ & $2.9(2.1-4.0)$ & 2.0 \\
\hline
\end{tabular}

RR: Relative risk, CI: Confidence interval, NNT: The number needed to treat or to harm. 


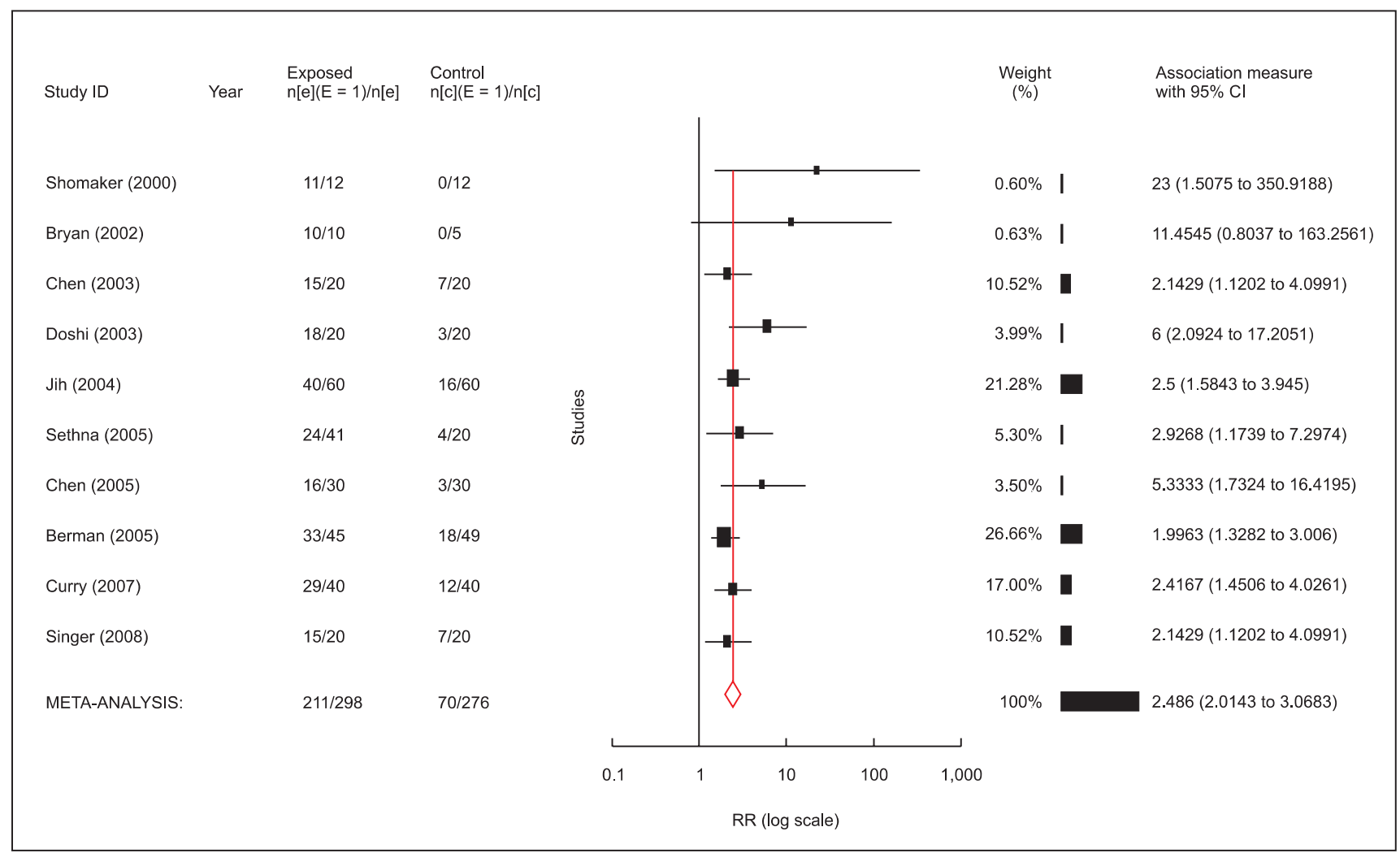

Fig. 1. An annotated forest plot (adequacy of cutaneous anesthesia) shows individual trials, depicted as filled squares, with the relative size of weights and horizontal bars as the confidence interval. The bottom diamond shape refers to the pooled value. RR: relative risk.

There were no severe adverse events and no trials presented any significant difference between the lidocaine/tetracaine patch or peel and placebo (Fig. 2). The lower limit of the 95\% confidence interval $(\mathrm{CI})$ was $0.9(\mathrm{OR}<1)$ that reported adverse effects showed no differences between the lidocaine/tetracaine medicated patch or peel and placebo. NNT for the adverse cutaneous effects was calculated as 14.9.

\section{Discussion}

Several methods are available to ease pain or discomfort for minor dermatologic procedures [28]. Application of the lidocaine/tetracaine medicated patch or peel to the skin 30 or 60 minutes before procedures may be an effective and safe means of anesthesia. Moreover, both the patch and peel formulation types provide convenient, noninvasive and painless means of application, with a rapid onset, minimal adverse effects, without the need for occlusion and easy removal $[19,22]$. Many clinical trials confirmed these potential advantages $[7,13,15,16,19,21$ 23]. However, a comprehensive literature analysis has not yet performed to test the hypothesis that the lidocaine/tetracaine medicated patch or peel is indeed an effective and safe method for reducing procedural pain. The present systemic review addressed the efficacy by comparing the patch or peel to placebo. Ten randomized, controlled trials were combined, and the data were statistically homogenous so a fixed effects model was used. Thus, we were able to draw a conclusion that the lidocaine/tetracaine medicated patch or peel was consistently and significantly more efficacious than placebo, based on subject-reported pain intensity, using the VAS scores.

A patch delivery system provides a specific amount of drug to a clearly distinct dermal area and is easier to apply and remove than the peel formula. Half the trials were with a patch formula, and the other half examined peel type applications. A transdermal patch included an oxygen-activated heating pad containing lidocaine and tetracaine $[7,22]$. This newlydeveloped drug delivery system uses controlled heat to enhance the delivery of local anesthetics through the skin. But the system was not found superior to the peel type application in this review. There was not much difference in efficacy and safety between subgroups of the lidocaine/tetracaine medicated patch and peel for dermal anesthesia over intact skin. Patches were administered for 20 minutes in 2 trials and for 30 minutes in 2 trials and for 39 minutes in 1 trial. For the peel type applications, they were administered for 30 minutes in 2 trials and for 60 minutes in 3 trials. Similar outcomes of RR and NNT might be 


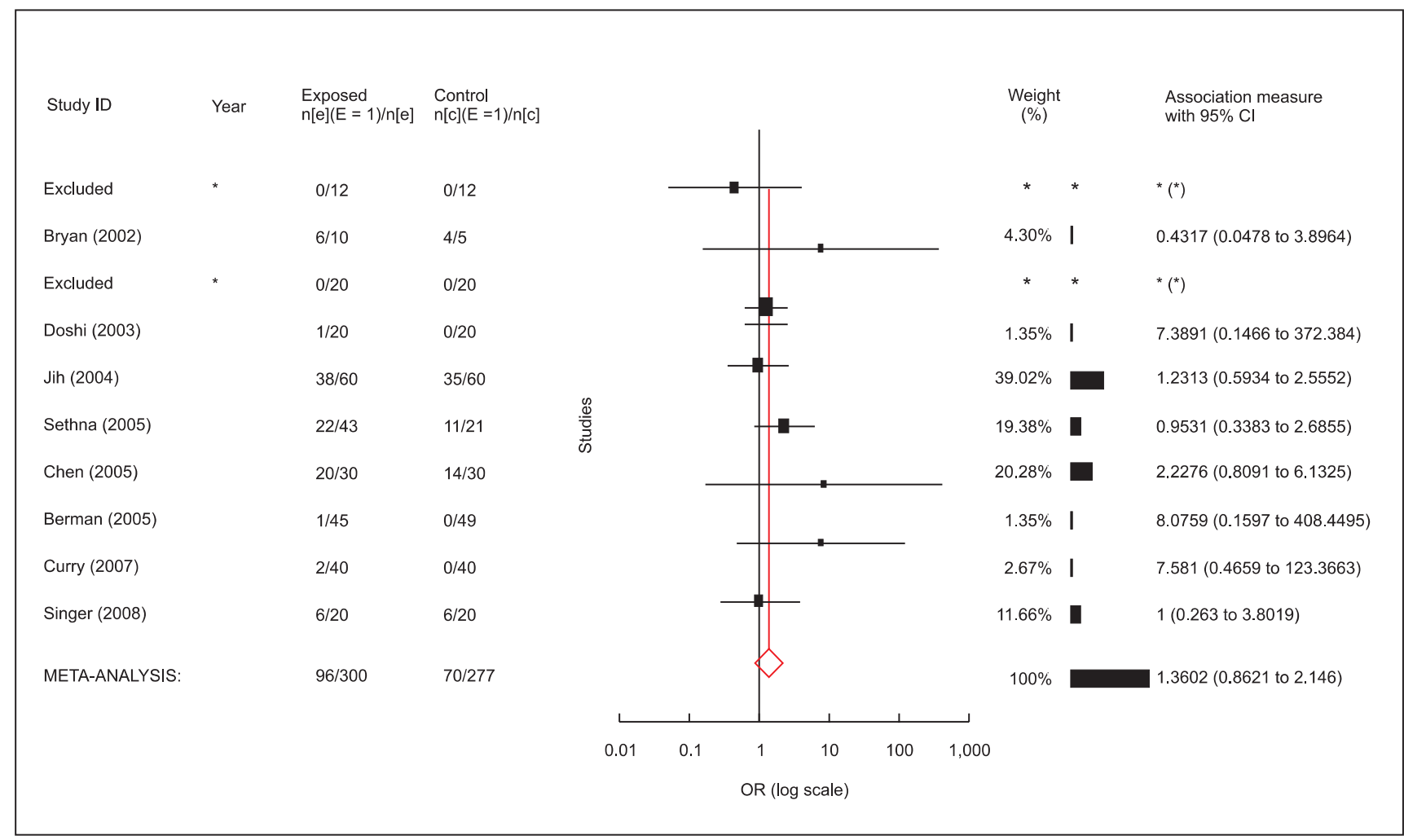

Fig. 2. An annotated forest plot (adverse effects) shows individual trials, depicted as filled squares, with the relative size of weights and horizontal bars as the confidence interval. The bottom diamond shape refers to the pooled value. OR: odd ratio.

attributed to the fact that the duration time of application was not considered in this review. It will be necessary to determine the impact of a heating component on the onset time with RCTs.

Adverse effects of the lidocaine/tetracaine medicated patch and peel were limited to transient mild erythema, blister, burning sensation, itching and edema. Even though the adverse effects were considered to be moderate in severity, they were resolved without intervention. Patch type formulas showed slightly more erythema and edema than placebo due to the vasodilating action of tetracaine and local heating system of the patch [9,21-23]. However, the difference in frequency was small so that the incidence of adverse effects of the medicated patch and peel was not significantly different from placebo, even when comparing 4 trials with the patch-type application. The potential for systemic absorption of tetracaine and lidocaine through intact skin is insignificant because blood concentrations of tetracaine and lidocaine were reported to be below the lower limit of quantitation $[8,18]$. The use of a medicated patch and peel was judged safe in this review.

There is a limitation of this study. Although we tried to minimize publication bias during arranging the process of review, there exist possible sources of error. Publication bias
(Egger's regression method, $\mathrm{P}<0.01$ ), the tendency of studies with positive results to be accepted as well as negative reports to be rejected, may alter the study outcome.

We concluded that the lidocaine/tetracaine medicated patch and peel were highly efficacious and safe in reducing local pain when applied 60 minutes prior to minor dermal procedures. Indeed, our results indicated that the patch and peel formula provided several potential clinical advantages such as minimal adverse effects and adequate dermal anesthesia in both adult and pediatric patients. Further trials are warranted to assess the effects of a heating drug delivery system on topical dermal anesthesia, prolongation of duration and reduction of the time of onset. Further, well-designed clinical trials are needed to compare the formulas with EMLA, liposome-encapsulated lidocaine or tetracaine, and BLT: triple anesthetic gel.

\section{References}

1. Eidelman A, Weiss JM, Lau J, Carr DB. Topical anesthetics for dermal instrumentation: a systematic review of randomized, controlled trials. Ann Emerg Med 2005; 46: 343-51.

2. Chen BK, Cunningham BB. Topical anesthetics in children: agents and techniques that equally comfort patients, parents, and clinicians. 
Curr Opin Pediatr 2001; 13: 324-30.

3. Croxtall JD. Lidocaine/tetracaine medicated plaster: in minor dermatological and needle puncture procedures. Drugs 2010; 70: 2113-20.

4. Shainhouse T, Cunningham BB. Topical anesthetics: physiology, formulations, and novel delivery systems. Am J Drug Deliv 2004; 2 : 89-99.

5. Tadicherla S, Berman B. Percutaneous dermal drug delivery for local pain control. Ther Clin Risk Manag 2006; 2: 99-113.

6. Friedman PM, Mafong EA, Friedman ES, Geronemus RG. Topical anesthetics update: EMLA and beyond. Dermatol Surg 2001; 27: 1019-26.

7. Shomaker TS, Zhang J, Love G, Basta S, Ashburn MA. Evaluating skin anesthesia after administration of a local anesthetic system consisting of an S-Caine patch and a controlled heat-aided drug delivery (CHADD) patch in volunteers. Clin J Pain 2000; 16: 200-4.

8. Alster TS. The lidocaine/tetracaine peel: a novel topical anesthetic for dermatologic procedures in adult patients. Dermatol Surg 2007; 33: 1073-81.

9. Kaweski S. Topical anesthetic creams. Plast Reconstr Surg 2008; 121: 2161-5.

10. Jadad AR, Moore RA, Carroll D, Jenkinson C, Reynolds DJ, Gavaghan DJ, et al. Assessing the quality of reports of randomized clinical trials: is blinding necessary? Control Clin Trials 1996; 17: 1-12.

11. Bax L, Yu LM, Ikeda N, Tsuruta H, Moons KG. Development and validation of MIX: comprehensive free software for meta-analysis of causal research data. BMC Med Res Methodol 2006; 6: 50.

12. Bax L, Yu LM, Ikeda N, Moons KG. A systematic comparison of software dedicated to meta-analysis of causal studies. BMC Med Res Methodol 2007; 7: 40.

13. Bryan HA, Alster TS. The S-Caine peel: a novel topical anesthetic for cutaneous laser surgery. Dermatol Surg 2002; 28: 999-1003.

14. Alster TS, Lupton JR. Evaluation of a novel topical anesthetic agent for cutaneous laser resurfacing: a randomized comparison study. Dermatol Surg 2002; 28: 1004-6.

15. Chen JZ, Alexiades-Armenakas MR, Bernstein LJ, Jacobson LG, Friedman PM , Geronemus RG. Two randomized, double-blind, placebo-controlled studies evaluating the S-Caine Peel for induction of local anesthesia before long-pulsed Nd:YAG laser therapy for leg veins. Dermatol Surg 2003; 29: 1012-8.

16. Doshi SN, Friedman PM, Marquez DK, Goldberg LH. Thirty-minute application of the S-Caine peel prior to nonablative laser treatment. Dermatol Surg 2003; 29: 1008-11.

17. Jih MH, Friedman PM, Sadick N, Marquez DK, Kimyai-Asadi A, Goldberg LH. 60-minute application of S-Caine Peel prior to 1,064 nm long-pulsed Nd:YAG laser treatment of leg veins. Lasers Surg Med 2004; 34: 446-50.

18. Sethna NF, Verghese ST, Hannallah RS, Solodiuk JC, Zurakowski D, Berde CB. A randomized controlled trial to evaluate S-Caine patch for reducing pain associated with vascular access in children. Anesthesiology 2005; 102: 403-8.

19. Chen JZ, Jacobson LG, Bakus AD, Garden JM, Yaghmai D, Bernstein LJ, et al. Evaluation of the S-Caine Peel for induction of local anesthesia for laser-assisted tattoo removal: randomized, doubleblind, placebo-controlled, multicenter study. Dermatol Surg 2005; 31: 281-6.

20. Schecter AK, Pariser DM, Pariser RJ, Ling MR, Stewart D, Sadick NS. Randomized, double-blind, placebo-controlled study evaluating the lidocaine/tetracaine patch for induction of local anesthesia prior to minor dermatologic procedures in geriatric patients. Dermatol Surg 2005; 31: 287-91.

21. Berman B, Flores J, Pariser D, Pariser R, de Araujo T, Ramirez CC. Self-warming lidocaine/tetracaine patch effectively and safely induces local anesthesia during minor dermatologic procedures. Dermatol Surg 2005; 31: 135-8.

22. Curry SE, Finkel JC. Use of the Synera patch for local anesthesia before vascular access procedures: a randomized, double-blind, placebo-controlled study. Pain Med 2007; 8: 497-502.

23. Singer AJ, Taira BR, Chisena EN, Gupta N, Chipley J. Warm lidocaine/tetracaine patch versus placebo before pediatric intravenous cannulation: a randomized controlled trial. Ann Emerg Med 2008; 52: 41-7.

24. George RB, Habib AS, Allen TK, Muir HA. Brief report: a randomized controlled trial of Synera versus lidocaine for epidural needle insertion in labouring parturients. Can J Anaesth 2008; 55: 168-71.

25. Sawyer J, Febbraro S, Masud S, Ashburn MA, Campbell JC. Heated lidocaine/tetracaine patch (Synera, Rapydan) compared with lidocaine/prilocaine cream (EMLA) for topical anaesthesia before vascular access. Br J Anaesth 2009; 102: 210-5.

26. Soltesz S, Dittrich K, Teschendorf P, Fuss I, Molter G. Topical anesthesia before vascular access in children. Comparison of a warmth-producing lidocaine-tetracaine patch with a lidocaineprilocaine patch. Anaesthesist 2010; 59: 519-23.

27. Masud S, Wasnich RD, Ruckle JL, Garland WT, Halpern SW, MeeLee D, et al. Contribution of a heating element to topical anesthesia patch efficacy prior to vascular access: results from two randomized, double-blind studies. J Pain Symptom Manage 2010; 40: 510-9.

28. Houck CS, Sethna NF. Transdermal analgesia with local anesthetics in children: review, update and future directions. Expert Rev Neurother 2005; 5: 625-34. 